\title{
Near-field optics and control of photonic crystals
}

\author{
A.F. Koenderink ${ }^{\mathrm{a}, 1}$, R. Wüest ${ }^{\mathrm{b}}$, B.C. Buchler ${ }^{\mathrm{a}}$, S. Richter ${ }^{\mathrm{c}}$, \\ P. Strasser ${ }^{\text {b }}$, M. Kafesaki ${ }^{d}$, A. Rogach ${ }^{\mathrm{e}}$, R.B. Wehrspohn ${ }^{\mathrm{f}}$, \\ C.M. Soukoulis ${ }^{\text {d,h,i }}$, D. Erni ${ }^{\mathrm{g}}$, F. Robin ${ }^{\mathrm{b}}$, \\ H. Jäckel ${ }^{\text {b }, ~ V . ~ S a n d o g h d a r ~}{ }^{\text {a,* }}$ \\ ${ }^{a}$ Laboratory of Physical Chemistry, Swiss Federal Institute of Technology (ETH), \\ CH-8093 Zürich, Switzerland \\ ${ }^{\mathrm{b}}$ Electronics Laboratory, Swiss Federal Institute of Technology (ETH), Gloriastrasse 35, \\ CH-8092 Zürich, Switzerland \\ ${ }^{\mathrm{c}}$ Max-Planck-Institute of Microstructure Physics, Weinberg 2, D-06120 Halle, Germany \\ ${ }^{\mathrm{d}}$ IESL, Foundation for Research and Technology Hellas (FORTH), P.O. Box 1527, \\ 71110 Heraklion, Crete, Greece \\ ${ }^{\mathrm{e}}$ Department of Physics and CeNS, Ludwig-Maximilians-Universität München, \\ D-80799 Munich, Germany \\ ${ }^{\mathrm{f}}$ Department of Physics, University of Paderborn, Warburgerstrasse 100, \\ D-33098 Paderborn, Germany \\ ${ }^{\mathrm{g}}$ Laboratory for Electromagnetic Fields and Microwave Electronics, \\ Swiss Federal Institute of Technology (ETH), Gloriastrasse 35, CH-8092 Zürich, Switzerland \\ ${ }^{\mathrm{h}}$ Ames Laboratory, Iowa State University, Ames, IA 50011, USA \\ ${ }^{\mathrm{i}}$ Department of Materials Science and Technology, University of Crete, Greece
}

Received 16 September 2005; received in revised form 20 September 2005; accepted 21 September 2005 Available online 11 October 2005

\begin{abstract}
We discuss recent progress and the exciting potential of scanning probe microscopy methods for the characterization and control of photonic crystals. We demonstrate that scanning near-field optical microscopy can be used to characterize the performance of photonic crystal device components on the sub-wavelength scale. In addition, we propose scanning probe techniques for realizing local, low-loss tuning of photonic crystal resonances, based on the frequency shifts that high-index nanoscopic probes can induce. Finally, we discuss prospects for on-demand spontaneous emission control. We demonstrate theoretically that photonic crystal membranes induce large variations in spontaneous emission rate over length scales of $50 \mathrm{~nm}$ that can be probed by single light sources, or nanoscopic ensembles of light sources attached to the end of a scanning probe.
\end{abstract}

(C) 2005 Elsevier B.V. All rights reserved.

PACS: 42.70Qs; 42.50.Pq; 42.25Fx

Keywords: Photonic crystal; Scanning near-field optical microscopy; Integrated optics; Cavity quantum electrodynamics; Spontaneous emission

* Corresponding author. Tel.: +41 446334621 ; fax: +41 446331316 .

E-mail address: vahid.sandoghdar@ethz.ch (V. Sandoghdar).

URL: http://www.nano-optics.ethz.ch

${ }^{1}$ Present address: FOM Institute for Atomic and Molecular Physics, Kruislaan 407, NL-1098 SJ Amsterdam, The Netherlands.

\section{Introduction}

It is widely expected that photonic crystals offer an advantageous solid state platform for cavity quantum electrodynamics, with the prospect of realizing highly 
miniaturized optical devices [1]. Great challenges accompany the promised control of light on subwavelength length scales. Apart from the need to develop fabrication techniques to sculpt semiconductor material with nanometer accuracy, one requires unconventional techniques for probing and manipulating the interaction of light with the photonic crystal structure. The usefulness of photonic crystal devices will be greatly increased if methods are available to locally and quickly tune or switch optical properties.

While standard optical techniques suffice to resolve the light scattering out of large devices, the diffraction limit prevents photonic crystal devices to be characterized beyond the resolution of about half a wavelength using conventional optics. A unique method to characterize the optical properties of photonic crystals that is not limited by diffraction is scanning near-field optical microscopy (SNOM) [2,3]. In SNOM, a sharp scanning probe in close proximity to the sample is used to image the optical field with a resolution that is only limited by the size of the probe and its separation from the sample. Thus, several groups have already applied this technique to asses the performance of photonic devices with resolutions around $100 \mathrm{~nm}$ [4-13]. In addition, we propose that scanning probe techniques used in SNOM may very well offer a convenient and efficient method to locally switch or tune optical devices in a controlled manner.

In this paper, we begin by describing how SNOM can be used to study the performance characteristics of a simple photonic crystal structure, with the particular example of a W3-W1 waveguide transition. In Section 3 , we discuss how scanning probe techniques can be used for controlling resonances in photonic crystals. Finally, in Section 4, we discuss the potential for obtaining on-demand, controllable changes in spontaneous emission dynamics with photonic crystals.

\section{Imaging photonic crystals with SNOM}

Although conventional imaging optics can successfully reveal some aspects of dispersion and loss in planar photonic crystal (PPC) [14], it is inherently a limited method. First, the relevant features of the electromagnetic field appear on the length scale of the lattice constant, which is comparable to the wavelength of light in the material. As high material indices $(\approx 3)$ are crucial for significant photonic crystal band gaps, the features that need to be imaged are smaller than the resolution of imaging optics [15] given by $\lambda / 2 \mathrm{NA}$, where NA denotes the numerical aperture of the imaging optics. The numerical aperture is particularly limited in photonic crystal applications because the use of an immersion medium would strongly perturb device operation. A second limitation of conventional microscopy is that it relies on propagating optical waves, which in the context of photonic crystals would require undesirable out-of-plane losses. Because SNOM detects non-propagating evanescent fields, it is not confronted with these problems [16]. Furthermore, in SNOM the optical data are measured simultaneously with the topography, allowing a very accurate assignment of optical features to the exact location on the structure. The advantages of SNOM as a high resolution imaging tool will be demonstrated in the following, using the example of a transition from a W3 waveguide (three missing rows of holes) to a W1 waveguide. Such transitions present potentially important building blocks in future highly integrated optical circuits [17].

Our measurement setup is depicted in Fig. 1(A) and consists of a collection SNOM with an uncoated, sharp fiber tip. Transverse electrically (TE) polarized light from a tunable laser $(\lambda=1480-1570 \mathrm{~nm})$ was coupled into the cleaved facet of a deeply etched trench access waveguide by an objective lens $(\mathrm{NA}=0.3)$. A large bend in the access waveguide ensures that the PPC is located away from the direct path of stray light coming from the incoupling region. To map the local electromagnetic field intensity, the tip is scanned along the

(A)
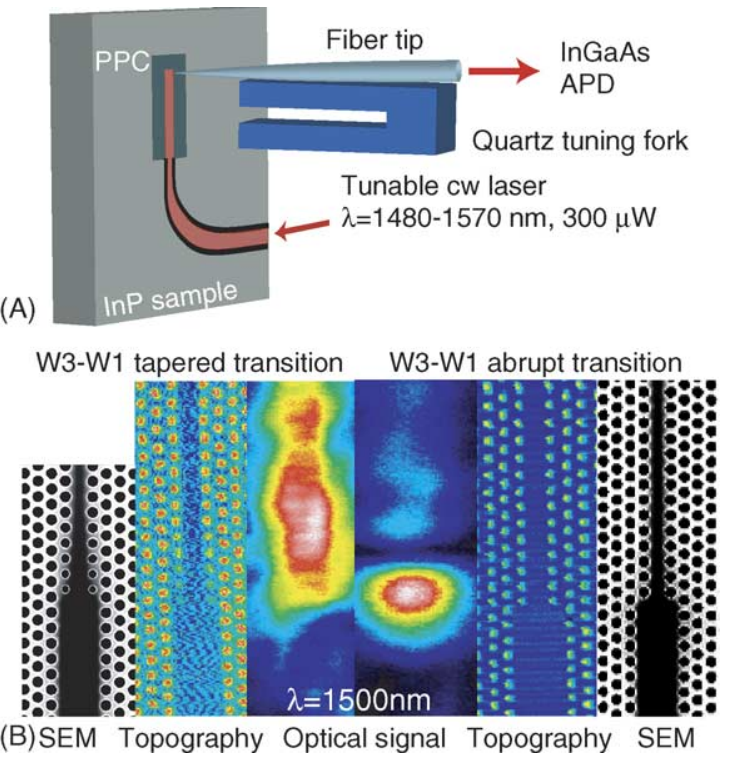

Fig. 1. (A) Schematic of the SNOM setup in collection mode. The tip scatters and collects evanescent and travelling electromagnetic waves, which are then detected with an APD photon-counting module. (B) SNOM measurements at $\lambda=1500 \mathrm{~nm}$ of a tapered (left) and an abrupt (right) W3-W1 transition together with the corresponding topography and SEM micrographs. 
sample surface. A tip-surface distance of $10-20 \mathrm{~nm}$ is maintained with the help of a quartz tuning fork using shear force feedback [18]. Radiation scattered by the tip is partially collected by the fiber itself and propagates to an InGaAs APD photon-counting module. In this way, topography (tip height) and optical signal of the PPC structure are simultaneously recorded.

Here, we show results for a structure that consists of a slab waveguide with a core of InGaAsP $(400 \mathrm{~nm}$, $n=3.43)$ and an upper InP cladding layer $(200 \mathrm{~nm}$, $n=3.17)$. The vertical slab waveguide supports one TE mode with an effective index of $n_{\mathrm{eff}}=3.29$ at $\lambda=1550 \mathrm{~nm}$. The PPC structure was etched through a slab waveguide and uses a triangular array of holes (depth $\approx 1.3 \mu \mathrm{m})$ with a lattice constant of $a=430 \mathrm{~nm}$. This results in a photonic bandgap for TE light for a range of reduced frequencies of $u=a / \lambda=$ 0.25-0.38. Further details on the sample and its fabrication can be found elsewhere [19,20].

Fig. 1(B) shows near-field measurements of a linearly tapered (hole radii: $0.24 a-0.375 a$ ) and of an abrupt transition from a W3 to $\mathrm{W} 1$ waveguide at $\lambda=1500 \mathrm{~nm}$ (middle plots). The color scale is normalized to maximize the contrast of each measurement. Next to the optical data the simultaneously acquired topography is displayed, which agrees well with the SEM micrographs (far right and left in Fig. 1(B)) of the fabricated structures. The optical data show that the two designs have a remarkably different effect on the flow of light through the transition region. The abrupt design exhibits strong out-of-plane scattering right at the waveguide transition and only a small part of the light is coupled into the $\mathrm{W} 1$ waveguide, whereas the taper manages to funnel the light efficiently into the $\mathrm{W} 1$ waveguide. We note that the signal level in W1 waveguides is generally much higher than in the W3 waveguides. This is due to a more concentrated mode profile and much higher out-of-plane losses for the W1 waveguide. Nevertheless, for both waveguides a characteristic spatial oscillation with a frequency of 1/a (see also Fig. 2) is evident. This feature is due to the interference of different Bloch components [6] and clearly demonstrates that sub-wavelength resolution is obtained in these measurements.

To gain further insight into our measurements, we compare them with simulations. Such simulations can only represent the structure correctly if the input parameters are known accurately enough. Due to fluctuations in fabrication, geometrical parameters like the normalized hole radius $r / a$ must be determined after fabrication in order to ensure the pertinence of the simulations. In recent work [21], we developed a

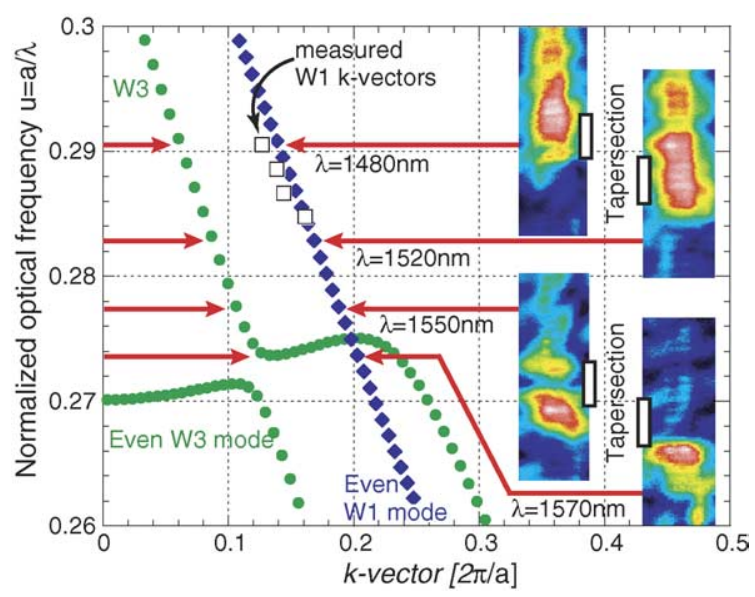

Fig. 2. Simulated dispersion relation of the two fundamental even modes of a W3 waveguide (green circles) and the one of a W1 waveguide (blue diamonds). Black open squares denote measured $k$ vectors of the W1 waveguide. On the right: optical data of SNOM measurements of the W3-W1 gradual taper transition (see Fig. 1(B) on the left) at different wavelengths. Red arrows indicate the corresponding frequency in the dispersion relation. The location of the taper section is indicated with a white bar. (For interpretation of the references to color in this figure legend, the reader is referred to the web version of this article.)

method to measure the wave vectors $k$ of light propagating in a $\mathrm{W} 1$ waveguide by deliberately setting up a standing wave. Wave vector measurements on the sample under investigation are shown as squares in Fig. 2 and were used together with $2 \mathrm{D}$ plane wave simulations [22] to determine the exact fill ratio $r / a=$ 0.375 for the W3-W1 transition that we characterize here. The slight offset between the measured $k$-vectors and the simulation in Fig. 2 is due to a $0.8 \%$ increase of material refractive indices at $\lambda=1480 \mathrm{~nm}$ compared to $\lambda=1550 \mathrm{~nm}$. Fig. 2 shows the dispersion relation of the two fundamental laterally even modes of the W3 waveguide and the one of the $\mathrm{W} 1$ waveguide calculated with a plane wave method [22] considering input parameters $r / a=0.375$ and $n_{\mathrm{eff}}=3.29$. For the frequency interval accessible with our laser system $(u=0.274-0.291)$, we expect to excite these even modes of the $\mathrm{W} 3$ and $\mathrm{W} 1$ waveguide. The modes show a linear dispersion with the exception of the W3 ministopband which appears in the simulation around a frequency of $u=0.272$. On the right-hand side we depict optical SNOM data measured at different wavelengths on the gradual W3-W1 taper. Red arrows indicate the respective positions of the measurements in the dispersion relation. The location of the taper section is marked with a white bar in the SNOM images.

It is expected that coupling from a W3 to W1 waveguide depends on the properties of the respective 
modes such as mode profile, $k$-vector and group velocity. The measurements in Fig. 2 show a clear dependence of the coupling properties on frequency. For frequencies clearly above the mini-stopband a strong signal inside the W1 waveguide was measured, indicating efficient coupling. As the wavelength increases and approaches the W3 mini-stopband, the coupling efficiency is drastically reduced and a region of strong scattering appears that shifts towards a location in front of the taper section. This suggests that the $\mathrm{W} 3$ mode is not well matched to the $\mathrm{W} 1$ waveguide mode, nor to the modes in the taper section at $\lambda=1570 \mathrm{~nm}$. The reason lies in the well-known fact that at wavelengths close to the mini-stopband the W3 mode exhibits a very slow group velocity (evidenced by the flat dispersion) which prevents coupling to the fast mode of the W1 waveguide.

The above-mentioned example shows the wealth of information that can be obtained using SNOM. By using coated fiber tips, the resolution can be further improved. However, it should be born in mind that higher resolution in SNOM also implies lower signal.

\section{Near field control of photonic devices}

In this section, we consider the use of scanning probe techniques to control optical devices, rather than characterizing them. Various methods have been suggested for tuning and switching. These include temperature tuning of the semiconductor refractive index [23], thermal or electromagnetic control of infiltrated liquid crystals [24,25] and ultrafast switching based on free-carriers or non-linearities such as the Kerr effect to tune the refractive index [26-29]. Recently, we have shown that another method that is not common in optics, but is widely used in the field of microwaves, is to shift the resonant frequencies of a structure by introducing a small dielectric perturbation in the near field of the mode [30]. By employing scanning probe techniques, it is possible to tune only a single unit in a structure containing many devices, as opposed to the global tuning obtained by other schemes. In addition, near-field control of photonic crystal devices has the advantage that the time scales for inducing, maintaining, removing and repeating the switching effect are completely independent of the properties of the materials constituting the photonic crystal.

As a case study, we have considered the tuning of microcavity resonances using near-field probes. Here, we will describe the results of three-dimensional finite difference time domain (FDTD) simulations $[31,32]$. In brief, we considered the tuning effect of dielectric cylindrical tips near membrane-type photonic crystals, consisting of a high index slab perforated with a hexagonal lattice (lattice constant, $a=420 \mathrm{~nm}$ ) and surrounded by air. We assumed a slab dielectric constant $\varepsilon=11.76$ typical for silicon, a membrane thickness of $250 \mathrm{~nm}$ and a hole radius $r=0.3 a$. Crystals over $5 \mu \mathrm{m}$ $\times 5 \mu \mathrm{m}$ in lateral size surrounded by up to $1 \mu \mathrm{m}$ of air were simulated, surrounded by Liao's absorbing boundary conditions. We used computational meshes with 14 or 20 grid points per $a$ parallel to the membrane, with twice the resolution normal to the membrane [32]. Grid-cell volume averaging of the dielectric constant was employed to reduce staircasing errors [33]. Quality factors and cavity mode frequencies were obtained by fitting a damped harmonic wave to time traces of the total $E$-field energy in the cavity.

The effectiveness of scanning probes for the local control of photonic crystal resonances is already shown in a very simple arrangement: we consider a photonic crystal membrane without any intentional defect. When a cylindrical tip is inserted into a hole in the hexagonal lattice of the photonic crystal membrane, it will essentially act as a point defect and create a low- $Q$ mode that is pulled down from the high-frequency band edge [34]. This switching configuration is relevant for the on-demand creation or tuning of transmission resonance filters with a $Q$ of $\sim 200$. As Fig. 3(A) shows, introducing a silicon tip $(\varepsilon=11.76)$ with a radius of $200 \mathrm{~nm}$ into a hole in a photonic crystal membrane indeed pulls down a resonance from the top of the gap. The frequency decreases linearly as the tip is pushed further into the membrane, but saturates once the tip protrudes all the way through the membrane. At this point, the quality factor of the resonance reaches its maximum of around 250. As Fig. 3(B) shows, the diameter of the tip sets the maximum tuning range, which is reached once the tip is completely inserted into the crystal. The maximum tuning range of half the gap width (as much as $150 \mathrm{~nm}$ ) is in theory obtained for a tip that completely fills an air hole [35]. As the resonance quality factor is limited to only about 250 , there is a minimum practical tip radius for which the shift of resonance away from the upper band edge exceeds its linewidth. For tip radii above $0.2 a$ a resonance is obtained that is clearly separated from the band edge, while the tip is still small enough to be easily manipulated in the air hole. It should be noted that this type of switching requires high index tips, such as silicon tips. Low index tips from materials such as glass do not provide sufficient index contrast to create resonances well separated from the band edge. In the context of SNOM characterization of photonic crystals, 


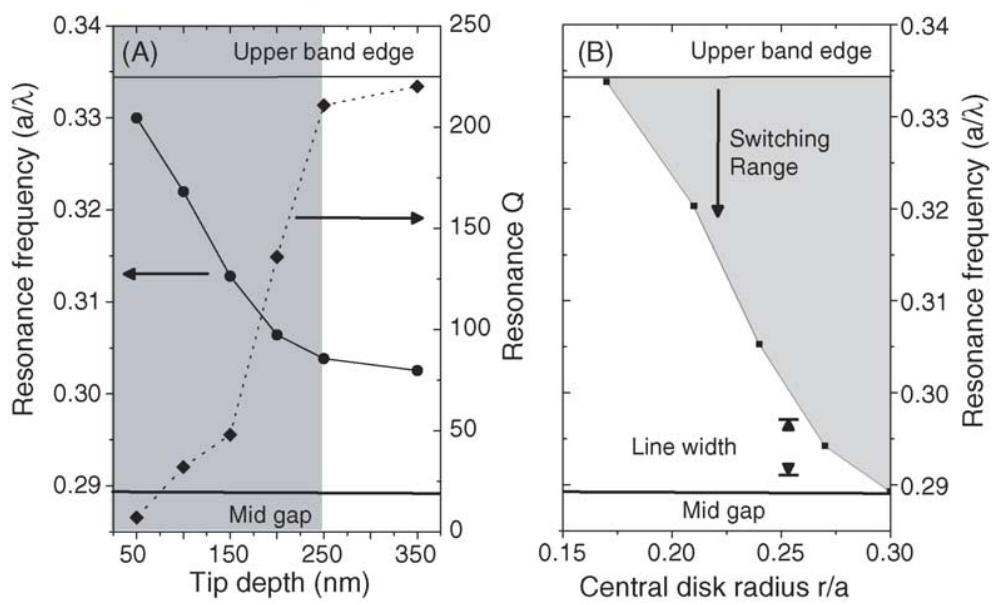

Fig. 3. When a silicon tip of radius $200 \mathrm{~nm}$ is inserted into a hole in a defect-free photonic crystal membrane (thickness $250 \mathrm{~nm}$, hexagonal lattice spacing $420 \mathrm{~nm}$, hole radius $0.3 a$ ), a low- $Q$ resonance is created. Panel (A) shows the calculated resonance frequency (circles, solid line, left-hand axis) and the quality factor (diamonds, dotted line, right-hand axis) as a function of the extent to which the tip is inserted into the membrane (indicated in gray). Panel (B) shows the calculated resonance frequency for fully inserted tips depending on the tip radius. The gray shaded area is the available switching range. The set of arrows shows the line width associated with this resonance that can be created on-demand.

this implies that glass tips do not significantly modify the mode structure of samples, as long as no strongly localized resonances are probed.

We have also studied the use of nanoscopic probes for the manipulation of cavities relevant for cavity quantum electrodynamics. Useful tuning of the coupling between the cavity and the quantum emitter requires that the resonance of a low volume cavity can be tuned over ranges of $10-100 \mathrm{GHz}$ without inducing large loss. We present results for a resonator that is formed about a defect of reduced radius $r=0.15 a$, and that is optimized in a manner similar to the method in Ref. [36]. By reducing the radius of two holes to $r=0.23 a$ on either side of the defect and then shifting them outwards by $0.11 a$, we create a non-degenerate dipole mode with a mode profile as shown in Fig. 4. The mode has a frequency $\omega a / 2 \pi c=0.284$ in the center of the 2D TE band gap, and the $Q$ is around 13,000, corresponding to a resonance linewidth of $15 \mathrm{GHz}$ at $\lambda=1500 \mathrm{~nm}$. This mode has the advantage of a small mode volume, at a quality factor that is high enough for useful tuning effects to be demonstrated, yet low enough to be easily simulated with FDTD. In addition, this type of cavity mode has a large amplitude in the central air hole, which is accessible to a near field probe. When a silicon tip is approached to the center of the cavity, we find that a significant frequency shift can be induced (cf. Fig. 5). As long as the tip is further than a few tens of nanometers from the photonic crystal membrane, we find that there is no significant loss. In this regime, the frequency shift increases exponentially as the tip is approached. Up to shifts of around $250 \mathrm{GHz}$, equivalent to $2 \mathrm{~nm}$ at $1500 \mathrm{~nm}$, low losses with $Q$ above 5000 can be maintained. This frequency shift is an order of magnitude larger than the cavity linewidth. The exponential increase of the induced detuning with approaching tip position matches the increasing overlap of the tip with the exponentially decaying evanescent mode profile of the cavity mode in the direction transverse to the photonic crystal membrane. For tip-membrane separations below $\sim 20 \mathrm{~nm}$, and once the tip is inserted into the central defect hole, the cavity quality factor drops below 5000,

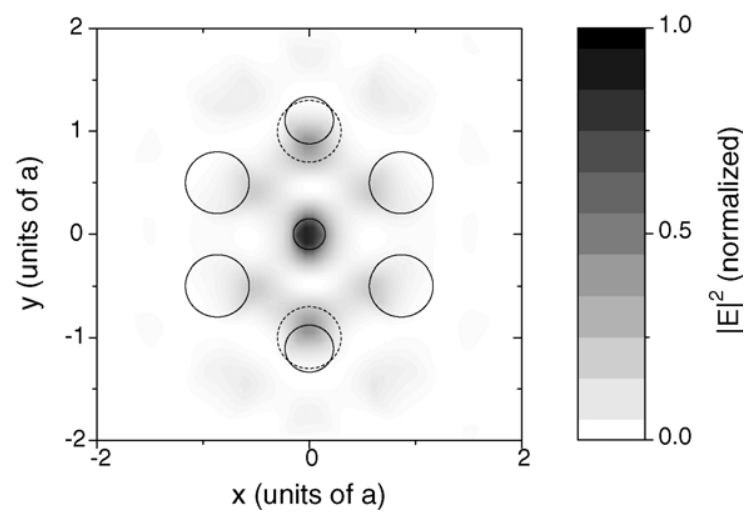

Fig. 4. Plot of the electric field intensity $|E|^{2}$ of the cavity mode for which we present lossless tuning predictions based on 3D FDTD. The cavity consists of a central defect (circle of reduced radius $0.15 a$ ) in a hexagonal lattice (spacing $a=420 \mathrm{~nm}$, hole radius $0.3 a$, thickness $250 \mathrm{~nm}$, dielectric constant $\varepsilon=11.76$ ) and is optimized by shifting and shrinking two holes (original holes dashed). 


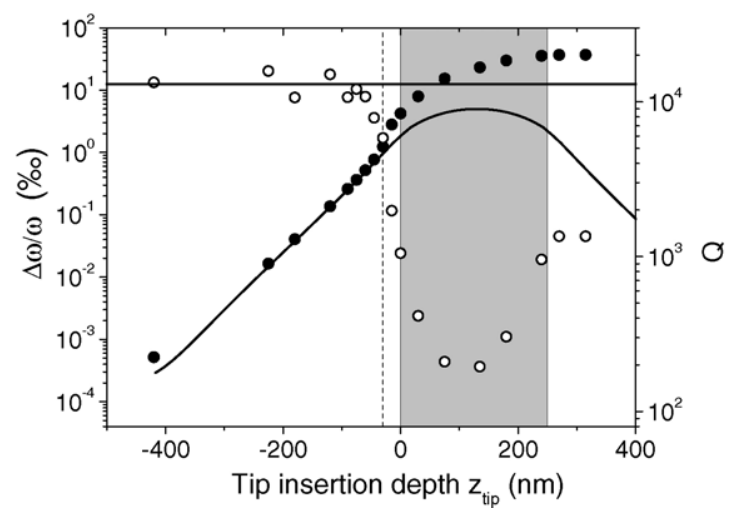

Fig. 5. Solid symbols show the relative induced frequency shift (lefthand axis) versus tip insertion depth calculated by 3D FDTD for the cavity in Fig. 4. The tip is centered above the central defect hole and $125 \mathrm{~nm}$ in diameter. Left of the gray area, which indicates the extent of the membrane, the tip apex is still away from the surface and constitutes a weak perturbation. The frequency shift follows the mode profile $|E|^{2}$ (solid curve). For tips up to $30 \mathrm{~nm}$ away from the membrane (vertical dashes) large $Q$-factors (open symbols, right-hand axis) can be maintained. The horizontal line shows the $Q$-factor in absence of the tip. The $Q$ drops when the tip apex is within the membrane and recovers when the tip extends all the way through the membrane.

to the level of about 200. For tips not fully inserted into the central defect, field profiles show strong scattering normal to the membrane on the side away from the tuning probe. However, once the probe fully extends through the slab the quality factor recovers to about 1000 .

The key to switching cavities without introducing a large loss is that the frequency shift increases linearly with the size of the perturber, while the loss contribution only grows quadratically with the perturbation. The magnitude of the perturbation can be measured by the polarizability of the tip volume that is inserted into the cavity $[37,38]$

$\alpha=3 \frac{\varepsilon-1}{\varepsilon+2} V_{\text {tip }}$ where $\quad V_{\text {tip }}=\pi r_{\text {tip }}^{2} d$,

where the tip volume corresponds to the tip crosssection $\pi r_{\text {tip }}^{2}$ times an effective height $d$ set by the exponential decay length of the mode into which the tip is inserted. Based on an exact theory originally developed for perturbations in microwave cavities, we find that the frequency shift is essentially limited by the ratio of the polarizability to the cavity mode volume [38,30]. Fig. 6 shows that the tuning effect predicted by FDTD for silicon tips indeed scales linearly with the tip cross-section $\pi r_{\text {tip }}^{2}$, while the loss induced by the tip scales quadratically with the tip area. Such quadratic loss is consistent with an interpretation of loss as Rayleigh scattering off the tip. Simulations of switching of different cavity designs and tip refractive indices confirm that the induced frequency shift is set by the ratio of polarizability to cavity mode volume, and also show that the quadratic scaling of loss with $\alpha$ allows to maintain a reasonably high $Q$ at large frequency shift [30].

As a caveat, we note that the simple relation between loss and the perturbation does not hold for all tip positions. For instance, we find that the induced loss strongly depends on the polarization of the mode at the position of the tip. For tip positions, where the electric field has a large contribution normal to the membrane and along the tip, light is scattered strongly into the tip [39]. Thus, there are positions where the induced frequency shift is small, in accordance with the low overall density of the mode at the tip position, but where large loss is induced due to the polarization selectivity of the near-field probe. On a more general note, the cavity loss is ultimately set by far-field interference encoded in the near-field profile of the cavity [36]. It has been shown that the loss of photonic crystal microcavities can be dramatically reduced by minute changes in the near-field mode profile that strongly affect the farfield interference $[36,40]$. In this light, it may even be possible to reduce losses of cavities by suitable placement of a perturbation.

In conclusion, we note that tuning by inserting probes into the near-field of an integrated optical device can be effective in general for dielectric structures that support resonances with mode volumes comparable to the polarizability of the perturber. For photonic crystals, cavities, waveguide bends, interferometers, directional

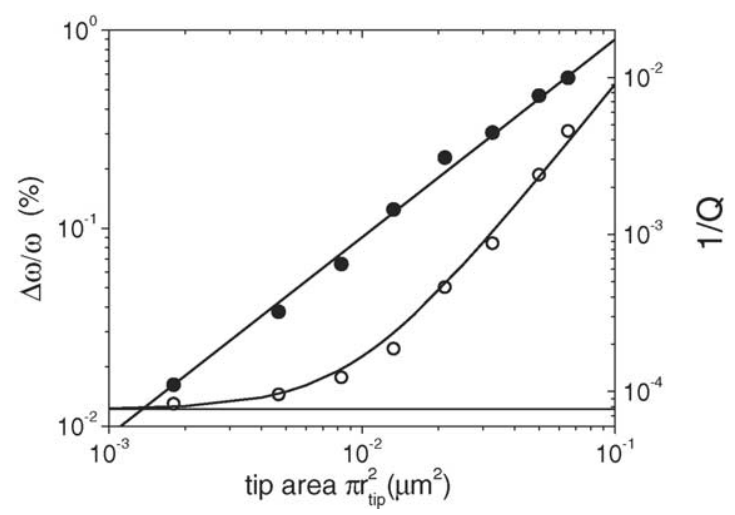

Fig. 6. Solid circles (left-hand axis) show the relative frequency shift induced by silicon tips $30 \mathrm{~nm}$ above the central defect hole in Fig. 4, as a function of the tip area $\pi r_{\text {tip }}^{2}$. The frequency shift is proportional to the tip area (fitted straight solid line). Open symbols show the concomitant induced loss $1 / Q$ (right-hand axis) which is set by the sum (fitted solid curve) of the $Q$ in absence of the tip (horizontal line) and a term that is quadratic in the tip area. 
couplers and filters can be tuned using this method. Finally, we remark that the discussion in this section could be directly extended to sensing applications if the sharp probe is replaced by a nanoscopic target object.

\section{Towards controlled spontaneous emission}

While several alternative routes towards small optical devices exist that do not rely on photonic crystals, complete control over spontaneous emission is an opportunity that is unique to photonic band gap materials. Complete inhibition of spontaneous emission can only be realized in three-dimensional band gap materials, but it is anticipated that large enhancements and reductions in spontaneous emission lifetime can be obtained also for sources coupled to two-dimensional photonic crystals. Unfortunately, several obstacles make the realization of spontaneous emission control extremely challenging. First, spontaneous emission control requires that an efficient light source is found that is compatible with the materials used for photonic crystals. Second, the spontaneous emission rate is expected to vary strongly with the position of the source in the unit cell via the spatial variation of the local density of modes [41]. Controlled experiments therefore require the use of single sources that are precisely controlled in position.

So far, experiments have largely relied on infiltration of dyes or nanocrystals that are deposited in random locations after fabrication of the crystals, or on the growth of random distributions of quantum dots during the fabrication process [42-47]. Badolato et al. have recently shown that it is possible to engineer the emittercrystal coupling, by first localizing the embedded quantum dots before etching the photonic crystal [48]. We propose a method that allows to control and vary the location of the light source after the photonic crystal fabrication. We envision a configuration in which the light source is attached to the tip of a scanning probe, as recently demonstrated in our group [49,50]. Such a configuration is ideal for the on-demand control of spontaneous lifetimes, simply by controlling the position of the tip. Furthermore, such a configuration potentially allows to keep the source separate from the photonic crystal backbone materials, thus avoiding surface-induced quenching of the emitter [51].

It is a particular challenge to realize spontaneous emission control in the near infrared region of 1.2$1.8 \mu \mathrm{m}$, in part due to the paucity of efficient emitters. We have recently identified mercury telluride nanocrystals (HgTe) as suitable candidates for the construction of subwavelength emitters in the infrared [52].

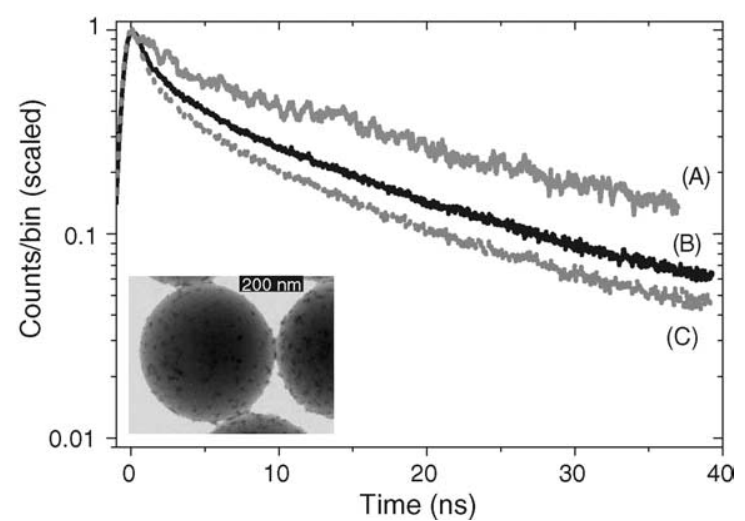

Fig. 7. Fluorescence decay traces of HgTe nanocrystals coated on latex spheres (curve (A), gray, taken from Ref. [52]), of nanocrystal deposit on the surface between cavities in Fig. 9 (B and C) (curve (B), black) and from nanocrystals coated inside a defect cavity in Fig. 9 (B and C) (curve (C), dotted gray). The inset shows a transmission electron microscopy image of a latex bead coated sparsely with $\mathrm{HgTe}$ nanocrystals. Curves were scaled to unity at $t=0$.

These nanocrystals have been reported to have fluorescence quantum efficiencies of around 50\% in water [53]. While detection of single nanocrystals remains elusive due to low quantum efficiencies and high dark count rates of infrared detectors, small colloidal particles down to $150 \mathrm{~nm}$ in size can be coated sparsely with quantum dots [54]. We have studied the photophysics of such fluorescent nanospheres (see Fig. 7 (inset)) spin-coated onto a cover slip, using confocal time-correlated single photon-counting fluorescence microscopy. Our measurements show that these spheres are efficiently excited with light at $532 \mathrm{~nm}$ and provide sufficient luminescence to facilitate their detection. Yet, these spheres remain as small as $\lambda / 10$ for light at $\lambda=1.5 \mu \mathrm{m}$, thus allowing their use as subwavelength luminescent probes. As shown in Fig. 7, the luminescence decay is close to single exponential, with a lifetime around $12 \mathrm{~ns}$, making these spheres suitable candidates for controlled cavity quantum electrodynamics experiments [52].

As a first experiment, where these HgTe quantum dot emitters are combined with photonic crystals, we have studied macroporous silicon photonic crystals [55] with selectively infiltrated dots [56]. These photonic crystals have a hexagonal lattice with lattice spacing $a=700 \mathrm{~nm}$ and a hole radius $R=0.45 a$. An in-plane band gap for TE polarized light is expected in the wavelength range $\lambda=1.4-2.3 \mu \mathrm{m}$. The crystals feature a superlattice of point defects, spaced by $5 a$ or $10 a$ (see Fig. 9(A)). Each point defect consists of a ring of six holes left unetched, around a central etched hole. The somewhat larger radius of the central defect hole, which 
is due to a proximity effect in etching the pores, allowed the selective infiltration of quantum dot emitters into the defects. To this end, a $\mathrm{SiO}_{2}$ layer was grown on top of the crystal. At a critical oxide thickness all pores are closed, except for a $100 \mathrm{~nm}$ opening above the larger central defect holes. These openings are sufficiently wide to infiltrate a mixture of polymer and $\mathrm{HgTe}$ dots to coat the inner surface of the defect hole. After infiltration, the oxide layer is removed by HF.

We have imaged these infiltrated point-defect structures using confocal microscopy in reflection mode (cf. Fig. 8). The green excitation $(\lambda=532 \mathrm{~nm})$ was focused onto the sample using a NA $=0.95$ objective that was also used to collect the infrared luminescence. As the objective was optimized for infrared, the divergence of the incident beam had to be carefully tuned in order for the excitation and detection focus to overlap on the sample surface. Raster scanning the sample provides an image of the infrared luminescence. The intensity of the reflected pump beam simultaneously reveals the topology of the sample. As shown in Fig. 9(B), the defect cavities as well as the underlying lattice are clearly resolved in the scattered light image. This is not unexpected since the lattice spacing far exceeds the diffraction limit at $532 \mathrm{~nm}$. A remarkable advantage of the setup, however, is that the resolution at infrared wavelengths is much better than the diffraction limit for the detected wavelength. It is set by the focus size of the pump beam.

In the scattered light image (Fig. 9(B)), the cavities appear as bright six-lobed rings, with a central dark spot. Here, it should be noted that the signal shows the

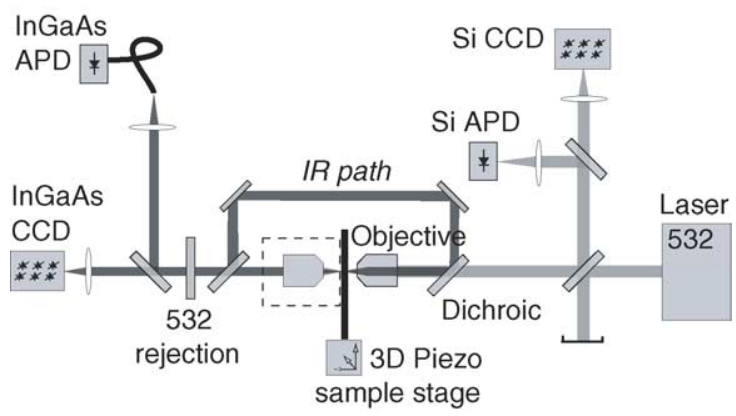

Fig. 8. Schematic of the infrared luminescence confocal setup used for Figs. 7 and 9. A laser beam ( $\lambda=532 \mathrm{~nm}$, picosecond pulses) is focused onto the sample with a microscope objective. A Si APD collects the reflected beam and acts as confocal aperture. The infrared luminescence is either collected in transmission (objective in dotted square) or in reflection via a dichroic mirror. For the infrared, the fiber core leading into the InGaAs APD (IdQuantique) acts as confocal aperture. Two CCD's at visible and infrared wavelengths are used for crude sample alignment. Images are recorded by raster scanning the sample that is mounted on a $3 \mathrm{D}$ piezo stage.
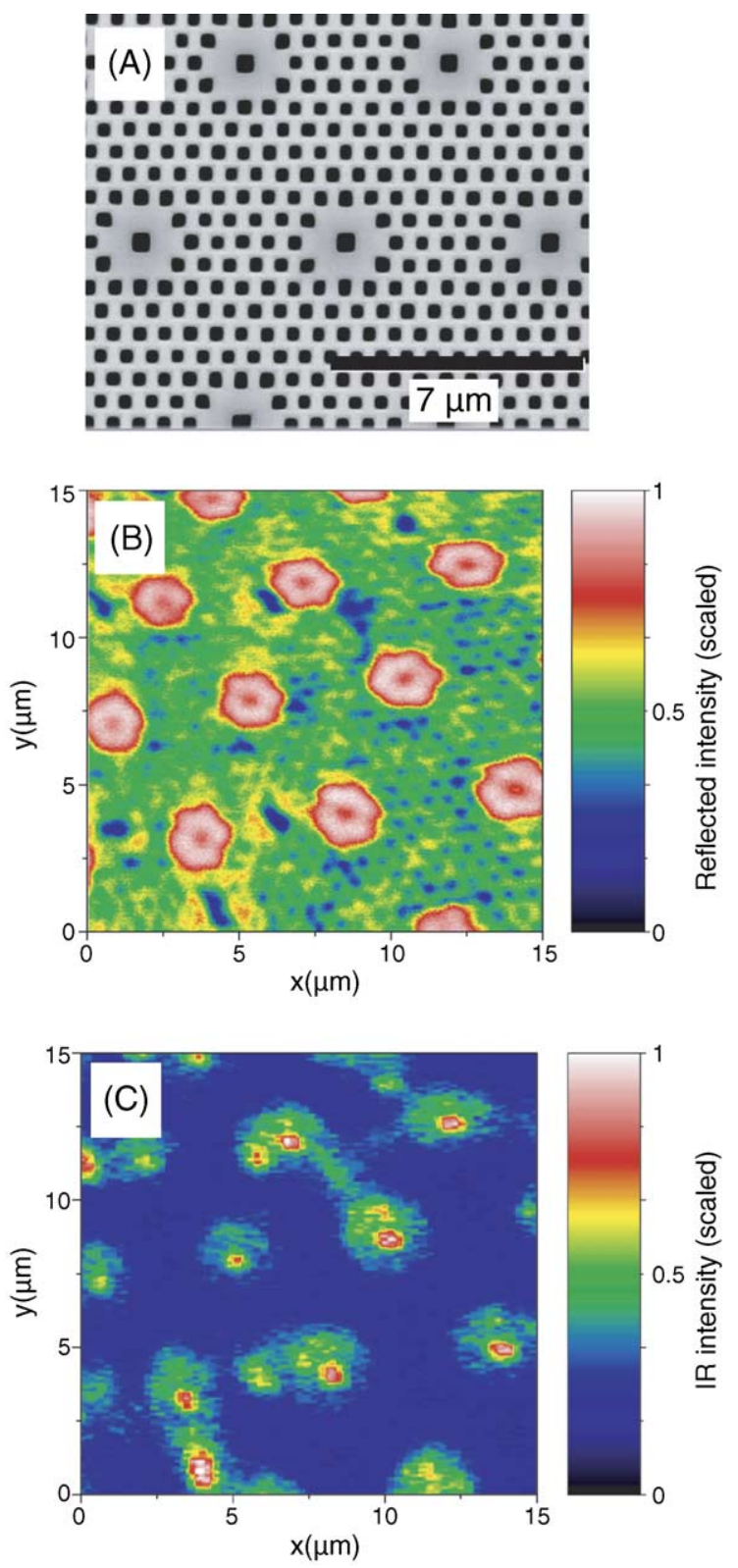

Fig. 9. Panel (A) shows a scanning electron micrograph of a macroporous silicon crystal (lattice spacing $a=700 \mathrm{~nm}$, hole radius $R / a=0.45$ ) with a superlattice of defects, each consisting of a ring of six unetched holes and a central defect hole. The scale bar is $7 \mu \mathrm{m}$. (B) Confocal microscopy scan of a $15 \mu \mathrm{m} \times 15 \mu \mathrm{m}$ area of such a crystal. A color bar for the collected reflected intensity at $532 \mathrm{~nm}$ (normalized) is shown on the right. The lattice spacing is easily resolved. (C) distribution of the infrared luminescence due to selectively infiltrated $\mathrm{HgTe}$ quantum dots taken in parallel with (B). The sample luminesces selectively from the defect cavities in (B). Bright luminescence centers that do not correspond to cavities can be traced to nanocrystal deposits that are evident as dark areas in (B). 
light reflected from the sample. The bright ring is hence explained by the stronger Fresnel reflection of unetched silicon, while the dark spot is due to the lack of reflection from the central air hole. The topology further shows a few dark patches apparently uncorrelated to the designed lattice. As discussed below, we attribute the reduced reflectivity at these positions to islands of (nanocrystal) material deposit which scatter the illumination light, hence reducing the reflection.

Fig. 9(C) shows the infrared luminescence collected in parallel with the rasterscan shown in Fig. 9(B). The infrared luminescence collected by the objective was detected by a fiber-coupled InGaAs avalanche photodiode (IdQuantique). As we do not detect single dots, the images represent luminescence over a wide spectrum from 1.3 to $1.7 \mu \mathrm{m}$, set by the size distribution of the HgTe dots. The luminescence image demonstrates that quantum dots have indeed been deposited preferentially inside the defect cavities, which show as bright dots on a background that is at the dark count level. The few isolated patches that appear dark in the topology also luminesce brightly, which validates their interpretation as clusters of $\mathrm{HgTe}$ nanocrystals on the surface of the sample. Close inspection of the cavities in the luminescence images shows that the cavities also luminesce when the excitation spot is located on the ring of unetched holes enclosing the central defect hole.

Using a time-correlated single photon-counting technique, we have for the first time been able to locally measure luminescence lifetimes from sources inside photonic crystal defect cavity structures with a spatial resolution better than the photonic crystal lattice constant. Fig. 7 shows a typical lifetime trace taken from a defect cavity. We note that the apparent emission time scale is in the range of $\sim 10 \mathrm{~ns}$, similar to the emission lifetime of HgTe dots in water or on the latex beads. This observation implies that the quantum dots do not suffer severe quenching and are hence promising light sources for probing emission lifetime modifications in silicon photonic crystals. However, we find that the spontaneous emission lifetime traces are not singleexponential. Similar non-single exponential decay, yet systematically slower is observed for the nanocrystal deposits that occur away from the cavities on the sample surface.

Such non-exponential emission decay dynamics and local variations in decay may have several origins, such as the presence of a non-radiative decay channel that is inhomogeneous over the spectral and spatial ensemble of dots that we probe, or density dependent dot-dot interactions, such as Förster energy transfer. Density dependent non-single exponential decay has indeed been observed for HgTe nanocrystals on latex beads with high nanocrystal coverages [52]. A third source of non-single exponential decay may be that the local density-of-states in the cavities is inhomogeneous over the ensemble of dots over which we integrate, which covers a wide spectrum extending over many coupled-defect cavity modes. To interpret the lifetime traces obtained from infiltrated samples in a quantitative manner, a full study must be performed that includes many samples with various controlled dot-densities, surface interactions and the use of a proper non-photonic reference environment [43]. To get around this problem, below we discuss a proposal for a non-contact experiment with a nanoscopic luminescent center that can be calibrated in absence of the sample.

We now turn to our efforts to identify what geometrical parameters for photonic crystal structures are suitable to create on-demand lifetime changes using scanning probe techniques. As scanning probe methods are inherently suited to probe the top surface of 2D photonic crystals, we concentrate on structures for which the relevant modes are located near the outer interfaces, rather than the deep-etched macroporous silicon crystals. We have therefore analyzed the spontaneous emission rate modifications that can be obtained with membrane photonic crystals [57]. The promising recent achievements in creating high- $Q$ photonic crystal cavities $[40,58,23,48]$ suggest that, although membranes have no 3D band gap, the vertical index confinement strongly enhances the role of the 2D band structure in threedimensional light control. Relevant questions that we seek to answer are if membranes cause significant lifetime changes, how far away from the membrane the source will still experience lifetime changes and how strong the lifetime variations are with lateral movement of the source through the 2D unit cell.

Due to the lack of symmetry of photonic crystal membranes, plane wave expansion methods [59-61] are not suited to calculate the local density-of-states (LDOS). Instead, we have used the three-dimensional FDTD method, as outlined above. As pioneered by Lee and co-workers [62,63], and as recently applied to photonic crystals by Hermann and Hess [64], the local density-of-states appearing in Fermi's Golden Rule for spontaneous emission [41] can be simulated using a classical dipole point source, that is excited with a given broadband pulse of current. The power spectrum of the work that the field emitted by the source does on the input current reveals the LDOS compared to vacuum after dividing by the work done on the same source embedded in a vacuum reference environment [62-64]. 


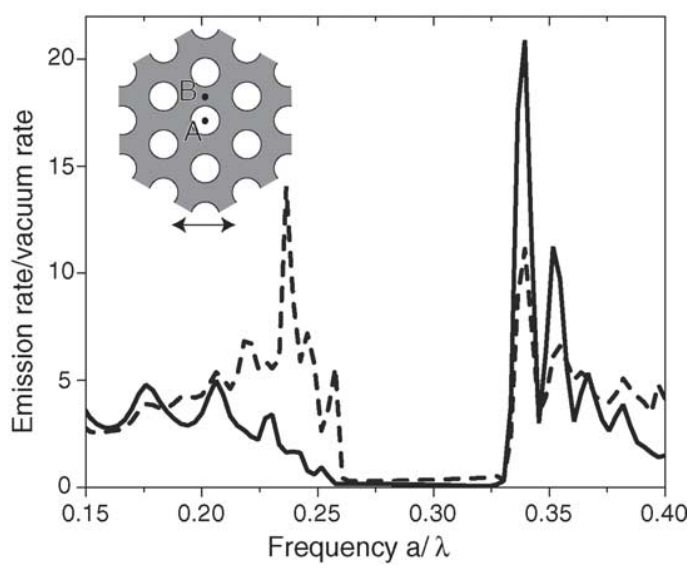

Fig. 10. Spontaneous emission rate modification (compared to vacuum) versus normalized frequency predicted by 3D FDTD for dipoles at two positions in the center plane of a photonic crystal membrane (thickness $250 \mathrm{~nm}$, hole radius $0.3 a$, with $a=420 \mathrm{~nm}$ the hexagonal lattice spacing). The solid curve corresponds to a dipole in an air hole and the dashed curve to a dipole centered in dielectric between two holes. Results are for dipole orientation as indicated by the arrow, and are calculated for a finite structure (hexagon of 13 holes across).

We focus on membranes of $250 \mathrm{~nm}$ thickness $(\varepsilon=11.76)$ containing air holes of radius $r / a=0.3$, where $a=420 \mathrm{~nm}$ is the lattice constant.
For dipoles oriented in the plane of the membrane we indeed find large variations in the spontaneous emission rates for frequencies around the in-plane TE band gap. Fig. 10 shows the modified emission rate compared to vacuum for a dipole centered in an air hole, and for a dipole midway between two air holes. These results were obtained for simulated crystal structures that were hexagons of 13 holes across, truncated by solid unperforated membrane extending into the absorbing boundary conditions. For low-frequencies, we find oscillatory behavior of the lifetime, due to Fabry-Pérot resonances associated with the total size of the simulated crystal structure. In the range $a / \lambda=0.26-0.33$, coincident with the TE band gap, the emission rate is strongly reduced. For the dipole in the air hole the reduction is about seven times compared to the vacuum emission rate. Large enhancements of the spontaneous emission rate occur around the band edge frequencies. Remarkably, emission occurs preferentially on the high-frequency edge for dipoles inside air holes, and preferentially on the lowfrequency edge for dipoles embedded in the semiconductor backbone. This is consistent with the well known concentration of high-frequency (low-frequency) 'air band' ('dielectric') modes in low (high)

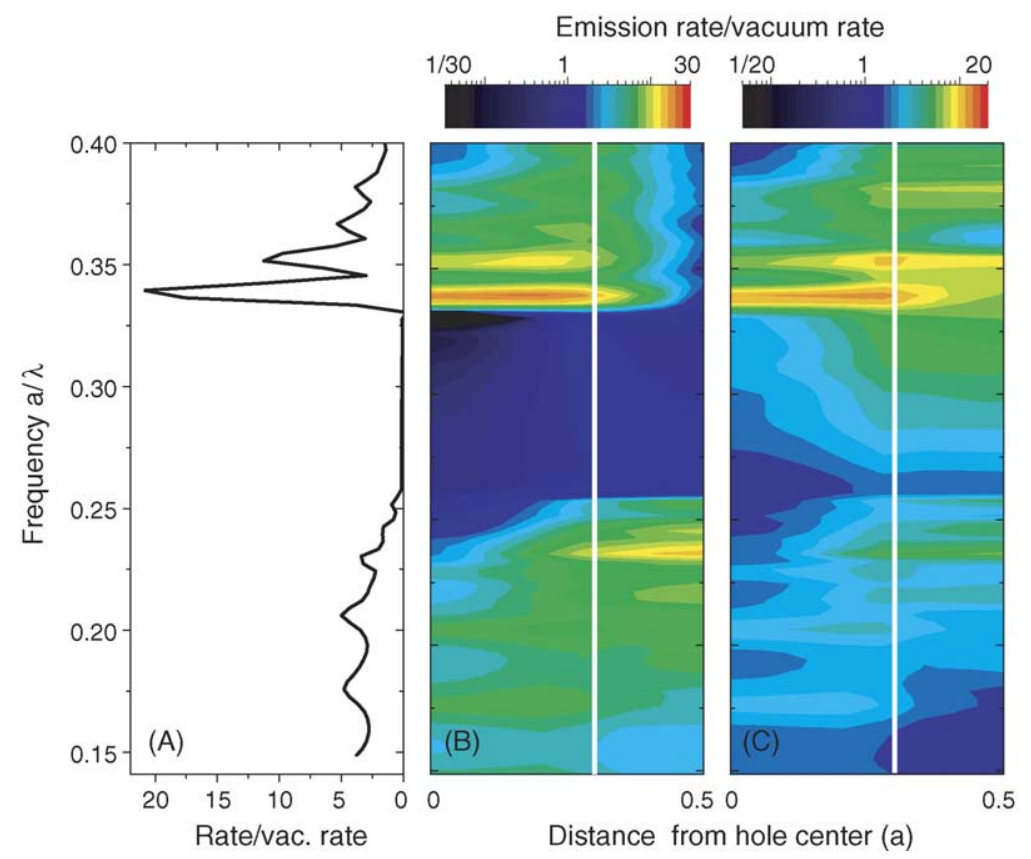

Fig. 11. Panel (A) again shows the lifetime enhancement compared to vacuum for a dipole centered in an air hole as a function of emission frequency (vertical axis, curve from Fig. 10). The contour plots in (B) and (C) show the lifetime modification (color scales shown on top) for dipoles in the midplane (B) and dipoles just above (C) the membrane as a function of frequency and of the lateral dipole position. The dipole position is changed from the center of an air hole to the mid-point between two holes. Evidently, there is a wide frequency range of emission inhibition that affects dipoles everywhere in the mid-plane of the membrane, and which coincides with the TE band gap. Above the membrane no significant inhibition remains, but the strong enhancements at the band edges persist. 
index material. The large band width of the emission rate inhibition attests to the strong effect of vertical index confinement in membranes: in structures that are not limited vertically, the local density-of-states reduction is much smaller, and does not occur over a wide bandwidth [65].

We have also assessed if these inhibitions and enhancements are only confined to the midplane of the membrane, or whether they persist up to the membrane edge [57]. To this end, we have calculated the emission lifetime changes as a function of frequency and as a function of lateral position in the unit cell for various dipole heights. In Fig. 11, results are shown for lateral dipole positions along a line connecting two air holes, starting from the center of a hole to the mid-point between holes. The results in (B) for a dipole in the mid-plane of the membrane confirms the results in Fig. 10. For all lateral dipole positions a frequency window of large inhibition occurs that coincides with the 2D band gap. Enhancements of the emission occur on the high- or low-frequency edge of the gap, depending on the position of the dipole. In Fig. 11(C), results are shown for dipole positions at the top surface just outside the membrane. This top surface is easily accessible using emitters attached to sharp near-field probes. Clearly, the large inhibition of emission is confined to the center of the membrane, and disappears at the membrane surface. At the band edge frequencies, however, large enhancements of up to 20 times the emission rate in vacuum remain. Moving the tip with the nanoscopic luminescent center laterally or vertically over length scales of just $50 \mathrm{~nm}$ causes large contrasts of a factor 10 to occur in emission lifetime. We have recently shown that the lifetime variations with distance to the membrane center can be understood in terms of the guided mode densities associated with dielectric slabs $[66,67]$.

\section{Acknowledgements}

For the results in Section 2, we acknowledge help with the simulations by Mario Agio and Rik Harbers. The samples described in Section 2 were fabricated in FIRST, the Center for Micro- and Nanoscale Science at ETH Zürich. We thank Wolfgang Stumpf for his contributions to the early stage of SNOM measurements. This work was carried out in the framework of the Network of Excellence ePIXnet, of the Swiss National Science Foundation program NCCR Quantum Photonics and the priority program SP 1113 of the Deutsche Forschungsgemeinschaft (DFG).

\section{References}

[1] C.M. Soukoulis (Ed.), Photonic Crystals and Light Localization in the 21st Century, Kluwer Academic Publishers, Dordrecht, 2001.

[2] D.W. Pohl, W. Denk, M. Lanz, Appl. Phys. Lett. 44 (1984) 651.

[3] M.A. Paesler, P.J. Moyer, Near-Field Optics: Theory, Instrumentation and Applications, Wiley-Interscience, 1997.

[4] P. Kramper, A. Birner, M. Agio, C.M. Soukoulis, F. Müller, U. Gösele, J. Mlynek, V. Sandoghdar, Phys. Rev. B 64 (2001) 233102.

[5] S.I. Bozhevolnyi, V.S. Volkov, J. Arentoft, A. Boltasseva, T. Søndergaard, M. Kristensen, Opt. Commun. 212 (2002) 51.

[6] S.I. Bozhevolnyi, V.S. Volkov, T. Søndergaard, A. Boltasseva, P.I. Borel, M. Kristensen, Phys. Rev. B 66 (2002) 235204.

[7] D. Gerard, L. Berguiga, F. de Fornel, L. Salomon, C. Seassal, X. Letartre, P. Rojo-Romeo, P. Viktorovitch, Opt. Lett. 27 (2002) 173.

[8] D.-J. Shin, S.-H. Kim, J.-K. Hwang, H.-Y. Ryu, H.-G. Park, D.-S. Song, Y.-H. Lee, IEEE J. Quantum Electron. 38 (2002) 857.

[9] P. Kramper, M. Kafesaki, C.M. Soukoulis, A. Birner, F. Müller, R. Wehrspohn, U. Gösele, J. Mlynek, V. Sandoghdar, Opt. Lett. 29 (2004) 174.

[10] E. Flück, M. Hammer, W.L. Vos, N.F. van Hulst, L. Kuipers, Photonics Nanostruct. 2 (2004) 127.

[11] K. Okamoto, M. Loncar, T. Yoshie, A. Scherer, Y.M. Qiu, P. Gogna, Appl. Phys. Lett. 82 (2003) 1676.

[12] H. Gersen, T.J. Karle, R.J.P. Engelen, W. Bogaerts, J.P. Korterik, N.F. van Hulst, T.F. Krauss, L. Kuipers, Phys. Rev. Lett. 94 (2005) 073903.

[13] H. Gersen, T.J. Karle, R.J.P. Engelen, W. Bogaerts, J.P. Korterik, N.F. van Hulst, T.F. Krauss, L. Kuipers, Phys. Rev. Lett. 94 (2005) 123901.

[14] M. Lončar, D. Nedeljković, T.P. Pearsall, J. Vučković, A. Scherer, S. Kuchinsky, D.C. Allan, Appl. Phys. Lett. 80 (2002) 1689.

[15] E. Abbe, Gesammelte Abhandlungen, vol. 2, G. Fischer Verlag, Jena, 1906.

[16] V. Sandoghdar, B.C. Buchler, P. Kramper, S. Götzinger, O. Benson, M. Kafesaki, Scanning near-field optical studies of photonic devices, in: K. Busch, S. Lölkes, R. Wehrspohn, H. Föll (Eds.), Photonic Crystals-Advances in Design, Fabrication, and Characterization, Wiley-VCH, Weinheim, 2004, pp. 215237.

[17] A. Talneau, P. Lalanne, M. Agio, C.M. Soukoulis, Opt. Lett. 27 (2002) 1522.

[18] K. Karrai, R.D. Grober, Appl. Phys. Lett. 66 (1995) 1842.

[19] P. Strasser, R. Wüest, F. Robin, C. Widmeier, D. Erni, H. Jäckel, in: Y. Matsushima (Ed.), 16th International Conference on Indium Phosphide and Related Materials, Conference Proceedings, vol. 16, 2004, pp. 175-178.

[20] R. Wüest, B.C. Buchler, R. Harbers, P. Strasser, K. Rauscher, F. Robin, D. Erni, V. Sandoghdar, H. Jäckel, in: G. Badenes (Ed.), Microtechnologies for the New Millennium 2005: Photonics and Optoelectronics, Proc. SPIE, vol. 5840, 2005, pp. 110-117.

[21] R. Wüest, D. Erni, P. Strasser, F. Robin, H. Jäckel, B.C. Buchler, A.F. Koenderink, V. Sandoghdar, R. Harbers, submitted for publication.

[22] S.G. Johnson, J.D. Joannopoulos, Opt. Express 8 (2001) 173.

[23] T. Yoshie, A. Scherer, J. Hendickson, G. Khitrova, H.M. Gibbs, G. Rupper, C. Ell, O.B. Shchekin, D.G. Deppe, Nature 432 (2004) 200. 
[24] K. Yoshino, Y. Shimoda, Y. Kawagishi, K. Nakayama, M. Ozaki, Appl. Phys. Lett. 75 (1999) 932.

[25] S.W. Leonard, J.P. Mondia, H.M. van Driel, O. Toader, S. John, K. Busch, A. Birner, U. Gösele, V. Lehmann, Phys. Rev. B 61 (1999) R2389.

[26] P.M. Johnson, A.F. Koenderink, W.L. Vos, Phys. Rev. B. 66 (2002) 081102.

[27] S.W. Leonard, H.M. van Driel, J. Schilling, R.B. Wehrspohn, Phys. Rev. B 66 (2002) 161102.

[28] A. Haché, M. Bourgeois, Appl. Phys. Lett. 77 (2000) 4089.

[29] T.G. Euser, W.L. Vos, J. Appl. Phys. 97 (2005) 043102.

[30] A.F. Koenderink, M. Kafesaki, B.C. Buchler, V. Sandoghdar, Phys. Rev. Lett. 95 (2005) 153904.

[31] A. Taflove, S.C. Hagness, Computational Electrodynamics: The Finite-Difference Time-Domain Method, second ed., Artech House, Boston, MA, 2000.

[32] M. Kafesaki, M. Agio, C.M. Soukoulis, J. Opt. Soc. Am. B 19 (2002) 2232

[33] O. Hess, C. Hermann, A. Klaedtke, Phys. Status Solidi A 197 (2003) 605.

[34] E. Yablonovitch, T.J. Gmitter, R.D. Meade, A.M. Rappe, K.D. Brommer, J.D. Joannopoulos, Phys. Rev. Lett. 67 (1991) 3380.

[35] P.R. Villeneuve, S. Fan, J.D. Joannopoulos, Phys. Rev. B 54 (1996) 7837.

[36] J. Vučković, M. Lončar, H. Mabuchi, A. Scherer, Phys. Rev. E 65 (2001) 016608.

[37] J.D. Jackson, Classical Electrodynamics, John Wiley \& Sons, New York, 1975.

[38] R.A. Waldron, Proc. Inst. Electron. Eng. 107C (1960) 272; For a review see O. Klein, S. Donovan, M. Dressel, G. Grüner, Int. J. Infrared Millim. Waves, 14 (1993) 2423.

[39] C. Girard, A. Dereux, O.J.F. Martin, M. Devel, Phys. Rev. B 50 (1994) 14467.

[40] Y. Akahane, T. Asano, B.S. Song, S. Noda, Nature 425 (2003) 944.

[41] R. Sprik, B.A. van Tiggelen, A. Lagendijk, Europhys. Lett. 35 (1996) 265.

[42] A.F. Koenderink, L. Bechger, H.P. Schriemer, A. Lagendijk, W.L. Vos, Phys. Rev. Lett. 88 (2002) 143903.

[43] P. Lodahl, A.F. van Driel, I.S. Nikolaev, A. Irman, K. Overgaag, D. Vanmaekelbergh, W.L. Vos, Nature 430 (2004) 654.

[44] S.P. Ogawa, M. Imada, S. Yoshimoto, M. Okano, S. Noda, Science 305 (2004) 227.
[45] M. Fujita, S. Takahashi, Y. Tanaka, T. Asano, S. Noda, Science 308 (2005) 1296.

[46] D. Englund, D. Fattal, E. Waks, G. Solomon, B. Zhang, T. Nakaoka, Y. Arakawa, Y. Yamamoto, J. Vučković, Phys. Rev. Lett. 95 (2005) 013904.

[47] A. Kress, F. Hofbauer, N. Reinelt, M. Kaniber, H.J. Krenner, R. Meyer, G. Böhm, J.J. Finley, Phys. Rev. B 71 (2005) 241304.

[48] A. Badolato, K. Hennessy, M. Atatre, J. Dreiser, P.M. Petroff, A. Imamoglu, Science 308 (2005) 1158.

[49] J. Michaelis, C. Hettich, J. Mlynek, V. Sandoghdar, Nature 405 (2000) 325.

[50] S. Kühn, C. Hettich, C. Schmitt, J.P.H. Poizat, V. Sandoghdar, J. Microsc. 202 (2001) 2.

[51] P.V. Kamat, Chem. Rev. 93 (1993) 267.

[52] P. Olk, B.C. Buchler, V. Sandoghdar, N. Gaponik, A. Eychmüller, A.L. Rogach, Appl. Phys. Lett. 84 (2004) 4732.

[53] A. Rogach, S. Kershaw, M. Burt, M. Harrison, A. Kornowski, A. Eychmüller, H. Weller, Adv. Mater. 11 (1999) 552.

[54] A.S. Susha, F. Caruso, A.L. Rogach, G.B. Sukhorukov, A. Kornowski, H. Möhwald, M. Giersig, A. Eychmüller, H. Weller, Colloids. Surf. A 163 (2000) 39.

[55] U. Grüning, V. Lehmann, C.M. Engelhardt, Appl. Phys. Lett. 66 (1995) 3254.

[56] S. Richter, M. Steinhart, H. Hofmeister, M. Zacharias, U. Gösele, N. Gaponik, A. Eychmüller, A. Rogach, S.L. Schweizer, A. von Rhein, R.B. Wehrspohn, J.H. Wendorff, Appl. Phys. Lett. 87 (2005) 142107.

[57] A.F. Koenderink, M. Kafesaki, C.M. Soukoulis, V. Sandoghdar, Opt. Lett., in press.

[58] K. Srinivasan, P.E. Barclay, O. Painter, J.X. Chen, A.Y. Cho, C. Gmachl, Appl. Phys. Lett. 83 (2003) 1915.

[59] T. Suzuki, P.K.L. Yu, J. Opt. Soc. Am. B 12 (1995) 570.

[60] K. Busch, S. John, Phys. Rev. E 58 (1998) 3896.

[61] R.Z. Wang, X.H. Wang, B.Y. Gu, G.Z. Yang, Phys. Rev. B 67 (2003) 155114.

[62] Y. Xu, R.K. Lee, A. Yariv, Phys. Rev. A 61 (2000) 033807.

[63] R.K. Lee, Y. Xu, A. Yariv, J. Opt. Soc. Am. B 17 (2000) 1438.

[64] C. Hermann, O. Hess, J. Opt. Soc. Am. B 19 (2002) 3013.

[65] D.P. Fussell, R.C. McPhedran, C.M. de Sterke, A.A. Asatryan, Phys. Rev. E 67 (2003) 045601.

[66] A.F. Koenderink, M. Kafesaki, C.M. Soukoulis, V. Sandoghdar, in preparation.

[67] H.P. Urbach, G.L.J.A. Rikken, Phys. Rev. A 57 (1998) 3913. 